\title{
Kinesin superfamily protein 17 contributes to the development of bone cancer pain by participating in NR2B transport in the spinal cord of mice
}

\author{
MING LIU, YUE LIU, BAILING HOU, DAN BU, LINYU SHI, \\ XIAOPING GU and ZHENGLIANG MA \\ Department of Anesthesiology, The Affiliated Drum Tower Hospital of Nanjing University Medical School, \\ Nanjing 210008, P.R. China
}

Received September 1, 2014; Accepted December 10, 2014

DOI: $10.3892 /$ or.2015.3706

\begin{abstract}
Treatment of bone cancer pain remains a challenge, while the mechanisms causing the pain remain elusive. We demonstrated that the expression of the N-methyl-D-aspartate (NMDA) receptor NR2B subunit was upregulated in mice with bone cancer pain. Kinesin superfamily protein 17 (KIF17), a recently characterized member of the kinesin superfamily proteins, has been demonstrated to transport and deliver the NR2B subunit to dendrites in mammalian neurons. In the present study, we induced bone cancer pain via femur bone cavity osteosarcoma NCTC 2472 tumor cell implantation (TCI) in mice. The results showed that TCI in mice increased the number of spontaneous flinches, mechanical allodynia events, expression of spinal KIF17 and NR2B subunits. Intrathecal administration of KIF17 antisense oligodeoxynucleotide (ODN) attenuated the behavioral signs of bone cancer pain and suppressed the increased expression of NR2B induced by TCI. In addition, KIF17 binds to a protein complex that contains mLin-10 to transport NR2B, and we determined that the increase of mLin-10 was suppressed following administration. Thus, these findings suggested that KIF17 contributed to the development of bone cancer pain in the spinal cord through NR2B transport and that mLin-10 may also play a role in pain development.
\end{abstract}

\section{Introduction}

A recent study predicted that given the current trends in population aging and growth, 22.2 million new cancer cases and 13.2 million cancer-related mortalities are expected

Correspondence to: Professor Zhengliang Ma, Department of Anesthesiology, The Affiliated Drum Tower Hospital of Nanjing University, No. 321 Zhongshan Road, Nanjing 210008, P.R. China E-mail: mazhengliang1964@nju.edu.cn

Key words: kinesin superfamily protein 17 , bone cancer pain, NR2B in 2030 (1). Although cancer pain may be present at any time during the course of the disease, it generally increases with disease progression. Thus, $75-90 \%$ of patients with metastatic or advanced stage cancer experience significant pain (2-4). The treatment of bone cancer pain continues to present a major clinical challenge since all the available therapies have significant unwanted side effects, and specific cell and molecular mechanisms, which may involve a combination of inflammatory and neuropathic pain, remain elusive $(5,6)$. The development of new analgesic therapies that are efficacious and have fewer side effects is of utmost clinical significance since it may improve patient quality of life and functional status.

NR2B, the most important N-methyl-D-aspartate (NMDA) receptor subunit, is intensively distributed at the site of transmission and regulation of pain, such as the spinal cord dorsal horn $(7,8)$. Central sensitization is an important mechanism in the development of chronic pain, and the NR2B subunit is substantially upregulated in the synaptic membrane, which is involved in the pain hypersensitivity caused by central sensitization (9-11). It was demonstrated that in mice with bone cancer pain, the expression of the spinal NR2B subunit was significantly increased, and it was thought important in the development of bone cancer pain (12). Prior to exerting its physiological role, newly synthesized NR2B must be transported into dendrites and localized to synapses, which is one of the prerequisites for NMDA receptor activity $(13,14)$.

Kinesin superfamily protein 17 (KIF17), a molecular motor expressed abundantly in mammalian neurons, has been proposed to exclusively bind to a protein complex that contains mLin-10 (Mint1/X11 $\alpha$, a synaptic-receptor sorting protein) and the NR2B subunit from the cell body to the dendrites of neurons $(15,16)$. Recent studies have focused on the cerebral cortex and the hippocampus and demonstrated that KIF17-based NR2B transport mechanisms for maintaining NR2A/2B levels may underlie multiple phases of memory processes in vivo (17). However, little is known regarding the precise roles and mechanisms of NR2B subunit transport at the spinal cord level in the mouse model of bone cancer pain.

In the present study, we determined that KIF17 is important in the process of bone cancer pain. Intrathecal administration 
of KIF17 antisense oligodeoxynucleotide (ODN) attenuated the pain behaviors in this model of bone cancer pain and decreased the expression of NR2B in mice. Furthermore, the expression of mLin-10 was suppressed. The results showed that in the spinal cord, KIF17 participates in the regulation of NR2B transshipment, which is involved in the development of bone cancer pain in mice, and mLin-10 may also be involved.

\section{Materials and methods}

Animals, drugs and administration. Animals were obtained from the Model Animal Research Center of Nanjing University. Sixty 4-6-weeks old adult male $\mathrm{C} 3 \mathrm{H} / \mathrm{HeJ}$ mice, weighing 20-25 g, were used in the present study. The mice were maintained in a temperature-controlled $\left(21 \pm 1^{\circ} \mathrm{C}\right)$ room with 12-h alternating dark/light cycles. The experimental protocols were approved by the Animal Care and Use Committee at the Medical School of Nanjing University. All efforts were made to minimize animal suffering and to reduce the number of animals used.

The sequences of the sense and antisense KIF17 ODNs were designed as previously reported (15). The sequences used were: sense, 5'-TTCGGTGGTGAGCCTCTG-3', and antisense, 5'-CAGAGGCTCACCACCGAA-3'. The ODNs were modified with phosphorothioate to improve stability and were purchased from Sangon Biotechnology Co. (Shanghai, China). The ODNs were dissolved in saline, and $5 \mu \mathrm{l}$ were injected intrathecally (i.t.) via lumbar puncture at the intervertebral space of L4-5 or L5-6. Injections occurred once per day for six consecutive days starting from day 14 after tumor cell implantation (TCI). KIF17 sense ODN (5 $\mu \mathrm{g})$, antisense ODN (5 $\mu \mathrm{g})$ and control saline $(5 \mu \mathrm{l})$ were injected i.t.

Murine model of bone cancer pain. TCI was achieved by injecting the osteosarcoma NCTC 2472, purchased from the American Type Culture Collection (Manassas, VA, USA; no. 2087787) into the intramedullary space of the right femur to induce bone cancer in mice. According to the ATCC recommendations, the tumor cells were cultured in NCTC 135 medium (Sigma-Aldrich, St. Louis, MO, USA) with $10 \%$ horse serum (Gibco, Grand Island, NY, USA). The cell incubator (Thermo Forma, Inc., Marietta, OH, USA) was kept at $37^{\circ} \mathrm{C}$ and had an atmosphere of $5 \% \mathrm{CO}_{2}$ and $95 \%$ air humidity. The cells were passaged twice per week.

The protocol described by Schwei et al was used to induce the murine model of bone cancer pain (18). The surgery was performed under anesthesia with pentobarbital sodium $(50 \mu \mathrm{g} / \mathrm{kg}$, intraperitoneally, i.p.). Gonarthrotomy was performed, exposing the femur condyles. A needle was employed to perforate the cortex and a hole was drilled using a dental bur. Twenty microliters of $\alpha$-minimal essence medium ( $\alpha$-MEM), containing $2 \times 10^{5}$ NCTC 2472 cells, were injected into the intramedullary space of the right femur to induce bone cancer pain. $\alpha$-MEM $(20 \mu l)$ with no cancer cells was injected in the sham surgery. The injection hole was subsequently plugged with a dental amalgam to tightly seal the injection hole in the bone and prevent the escape of tumor cells from the bone. The wound was irrigated with saline and then closed. The surgical operations were conducted under aseptic conditions.
Assessment of bone cancer-related pain behavior. The mice were randomly divided into the sham $(n=20)$ and TCI $(n=40)$ groups. On post-operation day 14, after the pain-associated behaviors were tested, the TCI mice were randomly divided into the TCI + saline group $(n=10)$, TCI + KIF17 sense ODN group $(n=10)$ and TCI + KIF17 antisense ODN group $(n=10)$. The number of spontaneous lifting actions and mechanical allodynia events was assessed in mice with bone cancer to evaluate bone cancer-associated pain. The tests were performed during the light phase; prior to each test, the mice were allowed to acclimatize for a minimum of $30 \mathrm{~min}$.

Spontaneous lifting behavior. The mice were placed in individual plexiglass compartments $(10 \times 10 \times 15 \mathrm{~cm})$. The behaviors of the mice were evaluated by measuring the number of spontaneous flinches of the right hind limb over a 2-min period of observation (19). Five periods were recorded for each mouse. Positive responses of the right hind limb involved a lifting movement and were not related to walking or grooming.

Mechanical allodynia test. Mechanical allodynia was measured using von Frey filaments (Stoelting, Wood Dale, IL, USA) with a protocol similar to that previously described (20). Briefly, the animals were individually placed beneath transparent plexiglass compartments $(10 \times 10 \times 15 \mathrm{~cm})$ on a metal-mesh floor (graticule: $0.5 \times 0.5 \mathrm{~cm}$ ). The minimum pressure was set to $0.16 \mathrm{~g}$, and the maximum pressure was set to $2.0 \mathrm{~g}$. The duration of each stimulus was $6-8 \mathrm{sec}$, and the interstimulus interval was a minimum of $15 \mathrm{sec}$. Brisk withdrawal of the paw and paw flinching were considered positive responses. The paw withdrawal mechanical threshold (PWMT) was determined by sequentially increasing and decreasing the stimulus strength (the 'up- and -down' method), which is the lowest von Frey filament that had three or more positive responses. Every mouse was tested five times per stimulus strength.

Western blot analysis. The spinal cord L3-5 segments were immediately removed from the deeply anesthetized mice (pentobarbital sodium, $50 \mu \mathrm{g} / \mathrm{kg}$, i.p.) and stored in liquid nitrogen. The tissue samples were homogenized in lysis buffer containing protease and phosphatase inhibitor. The samples were centrifuged at $12,000 \mathrm{rpm}$ for $10 \mathrm{~min}$ at $4^{\circ} \mathrm{C}$, and the supernatant was removed. The BCA method was used to determine the protein concentration (Kaiji Biotechnology, Nanjing, China). The samples were boiled at $100^{\circ} \mathrm{C}$ for $10 \mathrm{~min}$ with $1 \mathrm{X}$ loading buffer. Protein lysates $(50 \mu \mathrm{g})$ were separated using $10 \%$ SDS-PAGE and run for $30 \mathrm{~min}$ at $80 \mathrm{~V}$ and $90 \mathrm{~min}$ at $120 \mathrm{~V}$. The lysates were then transferred into polyvinylidene difluoride membranes (Millipore, Billerica, MA, USA) at $200 \mathrm{~mA}$ for $2 \mathrm{~h}$ (depending on the molecular weight of the target protein). The membranes were blocked in 5\% non-fat milk in Tris-buffered saline with Tween-20 (TBST) for $2 \mathrm{~h}$ at room temperature and then incubated with primary rabbit antibodies [anti-KIF17 (1:1,000), anti-NR2B (1:1,000), anti-mLin-10 antibody $(1: 1,500)$ all from Abcam, Cambridge, UK, and anti- $\beta$-actin $(1: 5,000)$ Cell Signaling Technology, MA, USA] diluted in blocking buffer. The following day, after washing six times with TBST for $1 \mathrm{~h}$, the membranes were incubated 

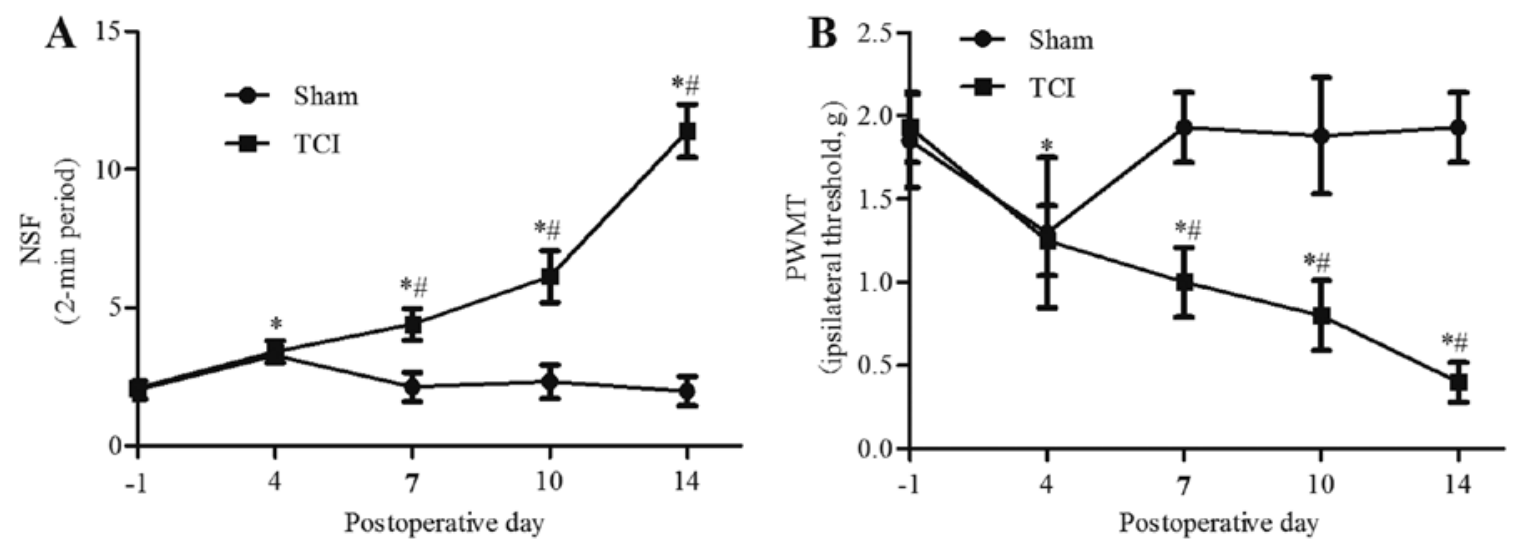

Figure 1. Time course of changes in the pain behavior after operation. (A) The number of spontaneous flinches (NSF) was increased after tumor cell implantation (TCI). (B) The paw withdrawal mechanical threshold (PWMT) was decreased after TCI. Data points are the means \pm SD. "P<0.05 vs. base; ${ }^{\text {"P }}<0.05$ vs. sham. Eight mice were included in each group.

with the secondary goat anti-rabbit antibody conjugated with horseradish peroxidase (1:5,000; Cell Signaling Technology) for $2 \mathrm{~h}$ at room temperature and then washed again for $1 \mathrm{~h}$. Immunoblots were detected using the ECL system (Millipore). The images of western blot analysis products were collected and analyzed using Quantity One V4.40 (Bio-Rad, Hercules, CA, USA). Each of the control and treatment groups was first standardized with $\beta$-actin.

Statistical analysis. Data are presented as the means \pm SD (standard deviation). The data were analyzed using repeated measures analysis of variance. The LSD post-hoc tests were performed to determine the sources of the differences when significant main effects were observed. $P$ values of $\leq 0.05$ was considered significant.

\section{Results}

Pain behavior over time. Prior to the operation, there was no significant difference between groups in the number of spontaneous flinches or in the PWMT $(\mathrm{P}>0.05)$. Compared with the baseline before the operation, on day 4 after the operation, the spontaneous flinches increased and the PWMT decreased in the TCI and sham groups $(\mathrm{P}<0.05)$. However, the pain behaviors in the sham group recovered to the baseline level at day 7. By contrast, TCI induced an increase of the

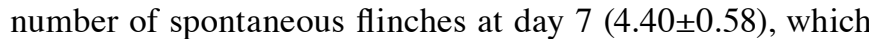
gradually increased over time until day 14 , when the P-value was $11.40 \pm 0.96$ (Fig. 1A). The PWMT decreased by day 7 $(1.00 \pm 0.21 \mathrm{~g})$, and on day 14 , the P-value was $0.40 \pm 0.12 \mathrm{~g}$ $(\mathrm{P}<0.05)$ (Fig. 1B).

Expression of KIF17, mLin-10 and NR2B in bone cancer pain. The results confirmed that in the sham and TCI groups, when compared with the baseline prior to operation, the expression of KIF17, mLin-10 and NR2B increased beginning on day 4 after the operation $(\mathrm{P}<0.05)$. However, the sham group recovered to the baseline level on days 7-14 $(\mathrm{P}>0.05)$. Compared with the sham group, the expression of KIF17, mLin-10, and NR2B in the TCI group was increased at day 7 and peaked on day 14 after the operation $(\mathrm{P}<0.05)$.
At day 14, the P-values were $1.77 \pm 0.10,1.64 \pm 0.27$ and 2.16 \pm 0.27 , respectively (Fig. 2)

Effect of the intrathecal administration of KIF17 antisense ODN on pain behavior in bone cancer pain. In the TCI group injected with saline and KIF17 sense ODN and the sham group injected with saline, the pain behaviors did not change, but remained at a regular level $(\mathrm{P}>0.05)$. Compared with the pre-lesion P-value prior to dosing and the TCI group injected with saline or KIF17 sense ODN, from 16 to 28 days after the operation, the spontaneous flinches were decreased and the PWMT was significantly increased in the TCI + KIF17 antisense ODN group $(\mathrm{P}<0.05)$. At day 16 , the $\mathrm{P}$-values were $3.38 \pm 0.47$ and $1.28 \pm 0.35 \mathrm{~g}$. At day 28 , the P-values were $8.98 \pm 1.15$ and $0.70 \pm 0.19 \mathrm{~g}$, respectively. However, at day 31, there was no significant difference between TCI groups in terms of the number of spontaneous flinches or the PWMT $(\mathrm{P}>0.05)$. Although injected KIF17 antisense ODN produced a significant transient inhibition of the spontaneous flinches and PWMT, compared with the sham + saline group, the difference remained significant $(\mathrm{P}<0.05)$ (Fig. 3).

Effect of the intrathecal administration of KIF17 antisense $O D N$ on mLin-10 and NR2B expression in bone cancer pain. We examined whether the effect of KIF17 antisense ODN during the development of bone cancer pain caused the reduction of NR2B protein within the spinal cord. Western blot analysis demonstrated that compared with the other TCI groups, the expression levels of KIF17, mLin-10 and NR2B in the spinal cord were significantly reduced following the application of KIF17 antisense ODN on day 19 and for the 6 days after intrathecal administration $(\mathrm{P}<0.05)$. Compared with the sham + saline group, the expression of KIF17, mLin-10 and NR2B remained upregulated $(\mathrm{P}<0.05)$. The $\mathrm{P}$-values were $0.86 \pm 0.05,0.82 \pm 0.07$ and $1.07 \pm 0.17$, respectively. No significant difference was identified between the saline group and the KIF17 sense ODN group for TCI (P>0.05) (Fig. 4). However, at 31 days after the operation, there was no significant difference among the three TCI groups in terms of the expression levels of KIF17, mLin-10 and NR2B in the spinal cord $(\mathrm{P}>0.05)$. The $\mathrm{P}$-values were $1.17 \pm 0.19$, $1.21 \pm 0.11$ and 1.12 \pm 0.09 , respectively (Fig. 5). 

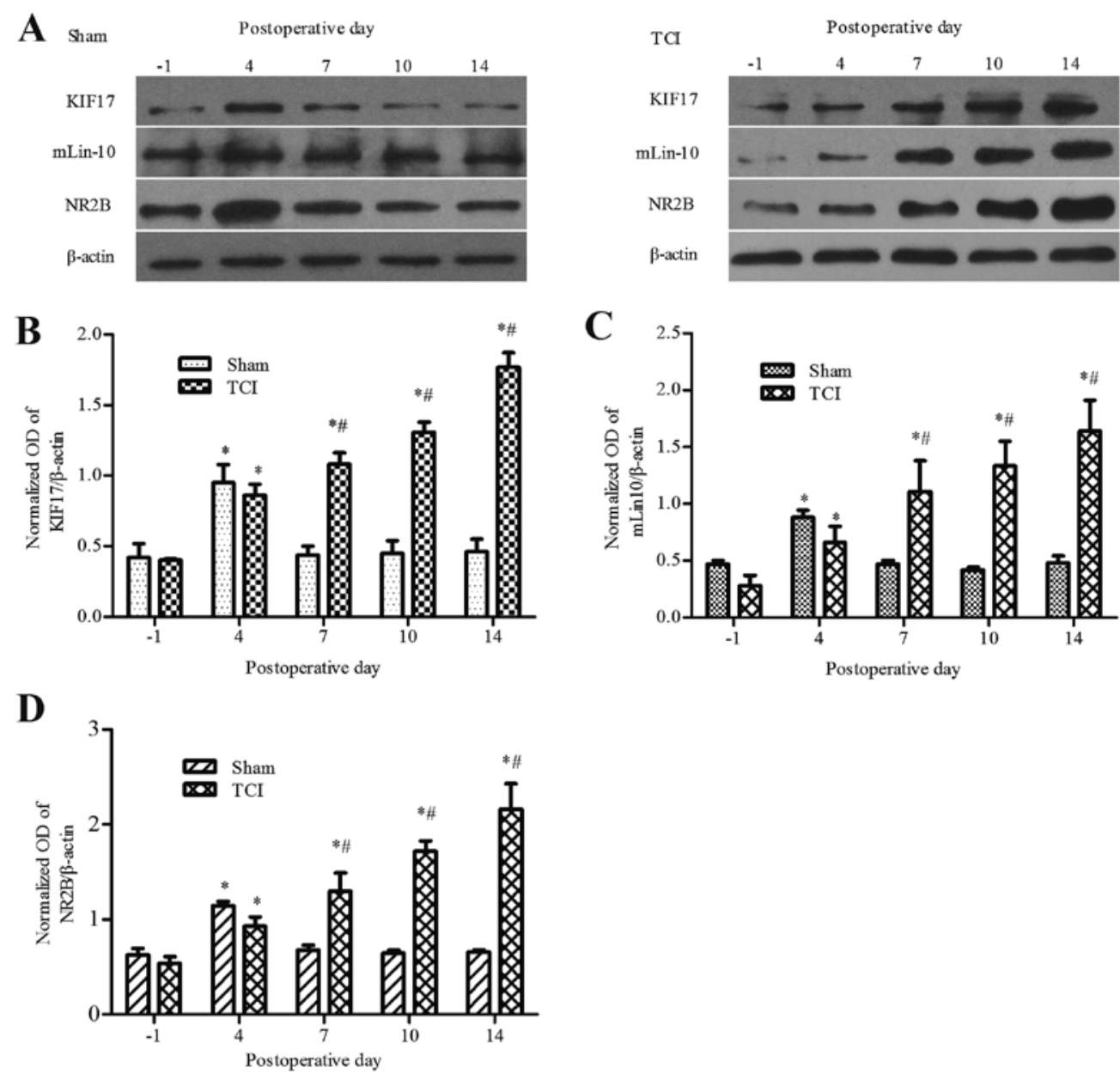

Figure 2. Upregulation of the expression of KIF17, mLin-10 and NR2B in the spinal cord after tumor cell implantation (TCI). (A-D) Examples of western blot analysis and data summary ( $\mathrm{n}=2$ per group) showing a time-dependent increased expression of the molecules. Data points are the means $\pm \mathrm{SD}$. ${ }^{*} \mathrm{P}<0.05 \mathrm{vs}$. base; ${ }^{\#} \mathrm{P}<0.05$ vs. sham.
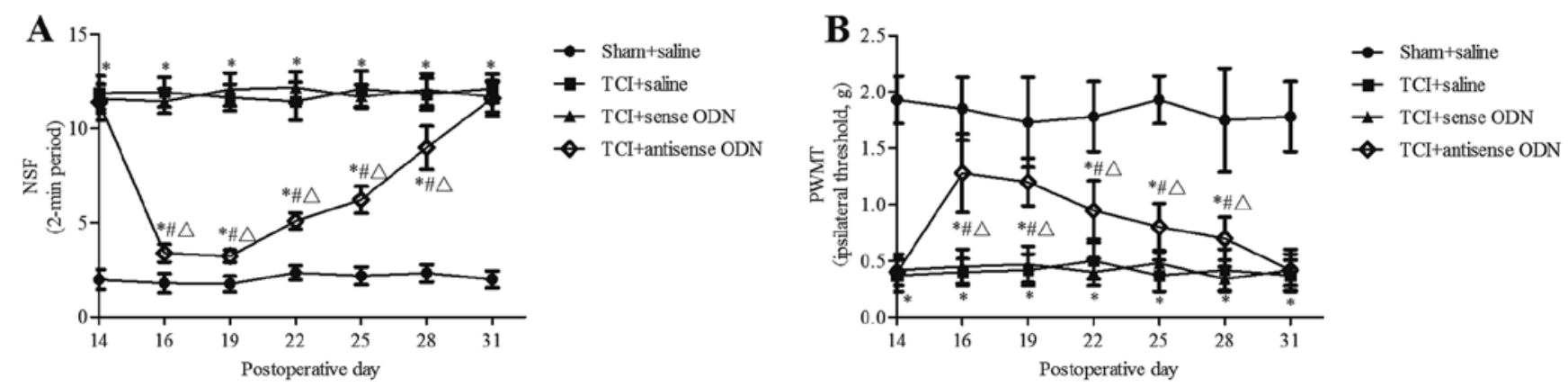

Figure 3. Time course of changes in pain behavior showing (A) the number of spontaneous flinches (NSF) and (B) the paw withdrawal mechanical threshold (PWMT) after intrathecal (i.t.) saline (5 $\mu \mathrm{l})$, KIF17 sense and antisense oligodeoxynucleotide (ODN) $(5 \mu \mathrm{g} / \mu \mathrm{l})$ on postoperative days 14-19 (once a day for six consecutive days). Data points are the means $\pm \mathrm{SD}$. ${ }^{*} \mathrm{P}<0.05$ vs. sham + saline; ${ }^{*} \mathrm{P}<0.05$ vs. the 14 days prior to dosing; ${ }^{\Delta} \mathrm{P}<0.05$ vs. TCI + saline and TCI + sense ODN. Eight mice were included in each group.

\section{Discussion}

KIF17 is a microtubule-based molecular motor that converts the chemical energy of ATP hydrolysis into a mechanical force used to transport cargo through microtubules (21). It has a pair of head motor domains that bind to microtubules, a coiled-coil stalk, and a tail domain that binds cargos $(22,23)$. In neurons, KIF17 participated in the transport of the NMDA receptor
NR2B subunit (15) along microtubules from the cell body to dendrites, which is considered to be the most important physiological effect of KIF17 and is associated with synaptic transmission, learning, memory and other functions.

The present study revealed the critical role of KIF17 in the process of bone cancer pain in mice. On day 4 after the operation, the pain behaviors and the expression of KIF17 and NR2B were altered in the TCI and sham groups, however, 
A
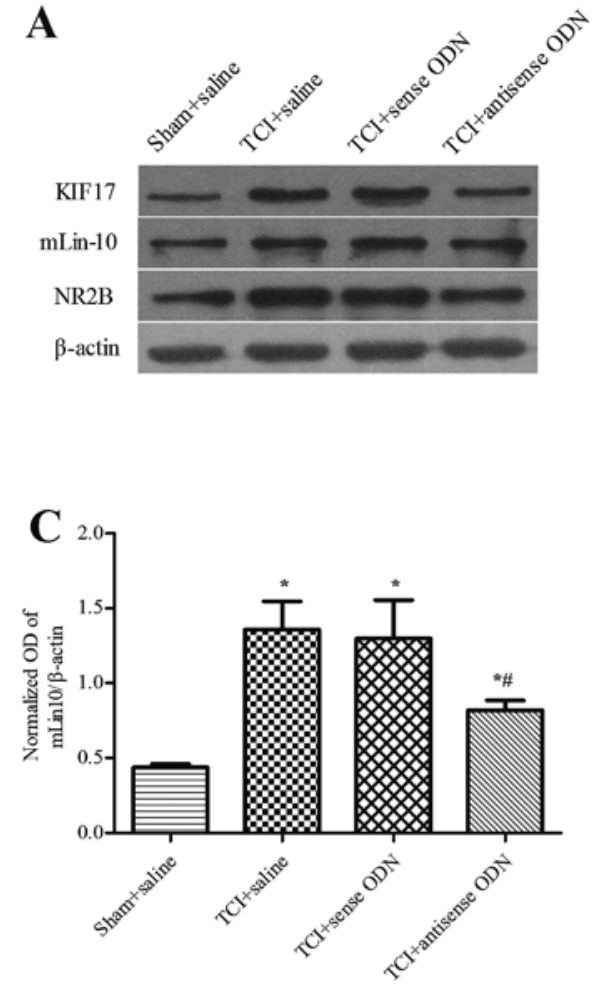
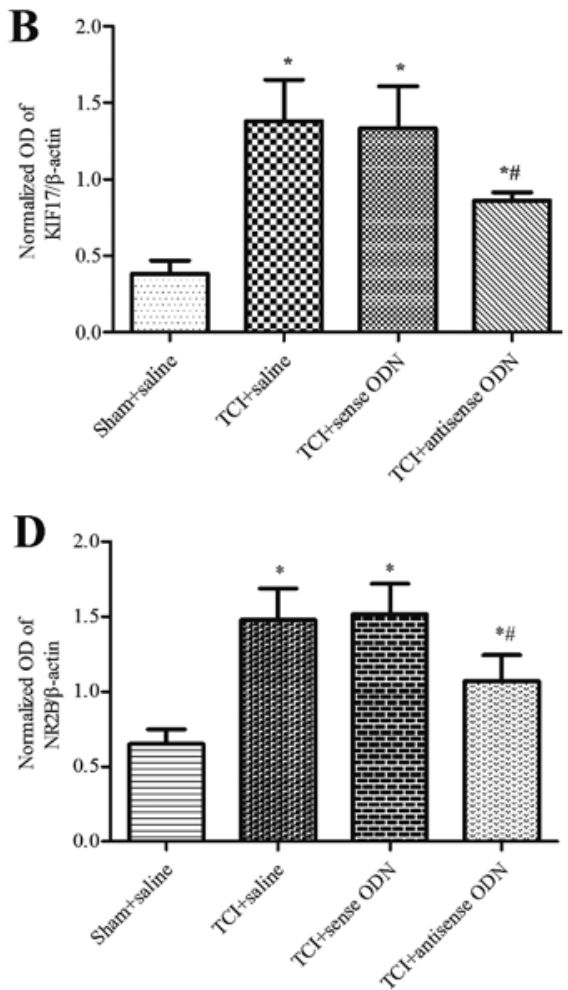

Figure 4. Downregulation of KIF17, mLin-10 and NR2B expression in the spinal cord after intrathecal administration of KIF17 antisense ODN (at 19 days after operation). (A-D) Examples of western blot analysis and data summary ( $\mathrm{n}=2$ per group). Data points are the means $\pm \mathrm{SD}$. ${ }^{*} \mathrm{P}<0.05$ vs. sham + saline; ${ }^{\#} \mathrm{P}<0.05$ vs. TCI + saline and TCI + sense ODN.
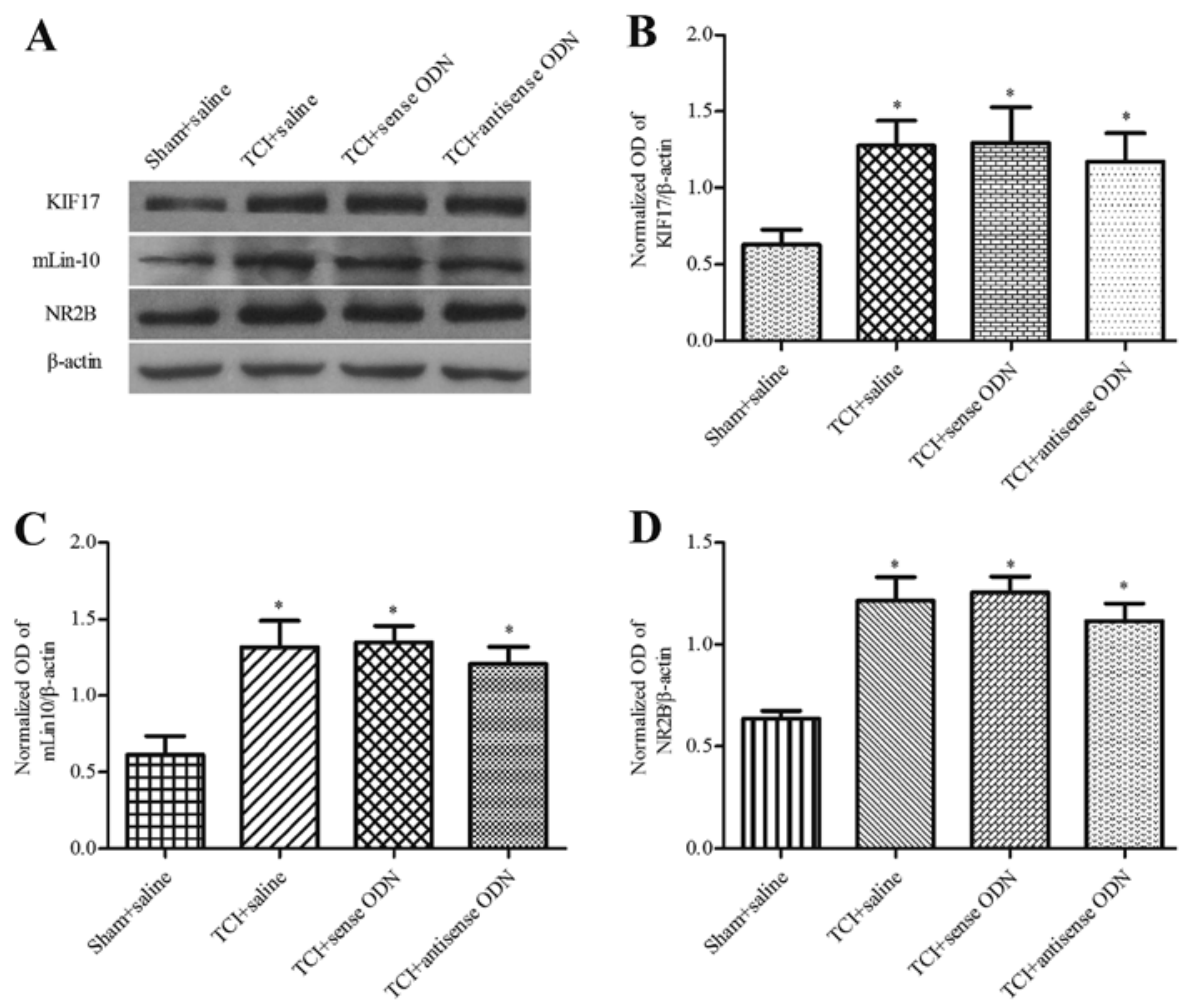

Figure 5. Upregulation of KIF17, mLin-10 and NR2B expression in the spinal cord at 31 days after operation. (A-D) Examples of western blot analysis and data summary ( $\mathrm{n}=2$ per group). Data points are the means \pm SD. $\mathrm{P}<0.05$ vs. sham + saline.

they were all recovered to the baseline level on day 7 in the sham group. These findings suggest that the pain behaviors and protein expression upregulation on day 4 were associated with the surgery and wound healing. In the TCI group, the 


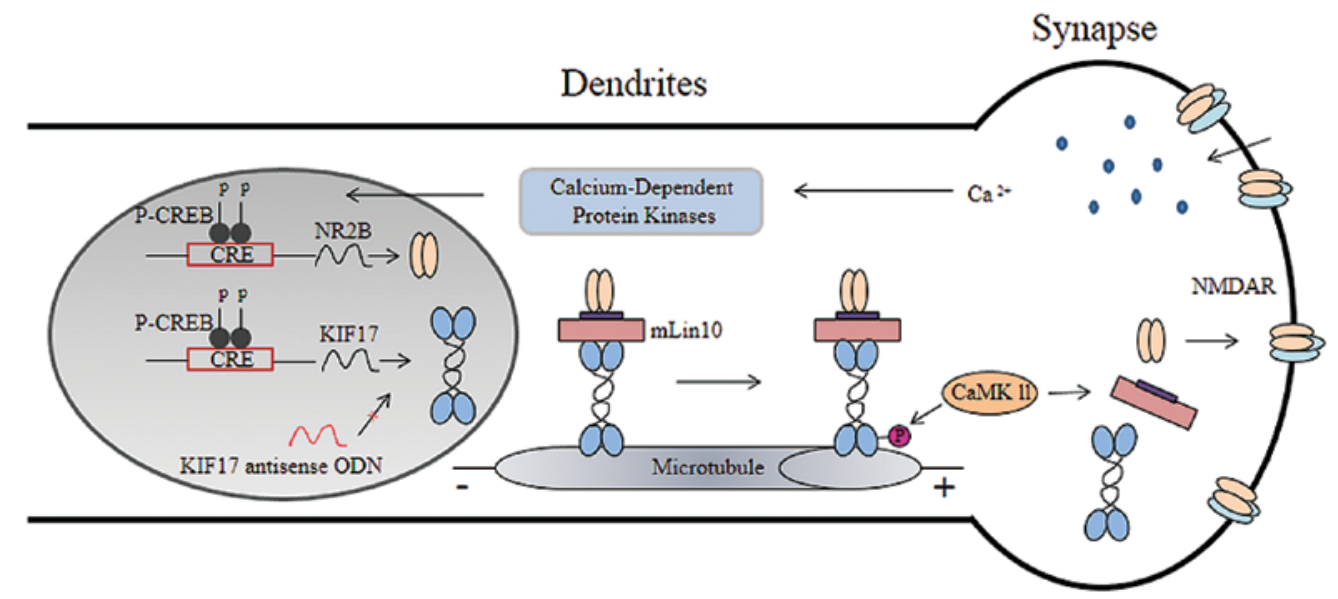

Figure 6. KIF17 binds to the scaffolding mLin-10 complex to transport NR2B from the cell body to synaptic terminals in dendrites.

number of spontaneous flinches was increased and the PWMT continuously decreased on days 7, 10 and 14. Simultaneously, the expression of KIF17 and NR2B in the spinal cord was significantly increased. Consistent with previous studies (?), the pain behaviors were most clear and stable at 14 days after the inoculation of bone cancer pain in mice. Our intrathecal administration was initiated on day 14 after the operation and occurred once per day for six consecutive days. During the period of continuous injection of antisense KIF17 ODN, the pain behaviors were clearly attenuated. Following the continuous dosing (at 19 days after the operation), the expressions of KIF17 and NR2B protein within the spinal cord was decreased. Thirty-one days after the operation, the analgesic effects disappeared, and KIF17 and NR2B expression levels were again upregulated.

The present study suggests that KIF17 transport of NR2B in the spinal cord may contribute to the development of bone cancer pain. When bone cancer pain occurs, the peripheral nociceptive receptors are activated by the noxious stimulation receptors, and the pain signals are then transferred to the spinal cord dorsal horn. This transmission results in increased excitatory neurotransmitter release on the presynaptic membrane, which results in the activation of calcium ion influx and calcium-dependent protein kinases. The kinases may also activate downstream of the substrate and ultimately increase the level of cAMP response element-binding protein (CREB). The KIF17 and NR2B gene promoter regions have the same CRE (TGACGTCA) sequence (24). Thus, the expression of KIF17 and NR2B increased after TCI. Then, NR2B was transported from the cell body. CaMKII-dependent phosphorylation of Ser 1029 is the molecular switch that triggers the release of NR2B from KIF17, thereby unloading NR2B to the dendrites (25), which forms the NMDA receptor and participates in the occurrence of bone cancer pain. Following the injection of antisense KIF17 ODN, the expression of KIF17 was decreased and the inhibition of KIF17 transported NR2B. The NR2B on the dendritic membrane decreased, and the pain behaviors of the mice were suppressed.

Previous studies considered that the transshipment of NR2B was accomplished via a direct interaction of the KIF17 tail domain with a PDZ domain of mLin-10 (Mint1/X11 $\alpha)(26,27)$.
mLin-10 is a shared component of the polarized protein localization pathway in neurons and epithelia and is detectable only in neurons. It comprises of a variable N-terminal region and a constant $\mathrm{C}$-terminal region that contains two PDZ domains. One domain binds to KIF17, and the binding tail is necessary for this function of KIF17 (26). The binding was very specific between the KIF17 tail domain and the first PDZ domain of mLin-10. The disruption of the first PDZ domain decreased the binding with KIF17, whereas the disruption of the second PDZ domain or the deletion of the phospholipid interaction (PI) domain did not alter the interaction (26). Similarly, it has been demonstrated that the intrathecal administration of selected peptide Myr-RC-13 perturbs the spinal KIF17/mLin-10 interaction and attenuates bone cancer pain (28).

The expression of mLin-10 in the spinal cord in bone cancer pain was assessed. We determined that the expression of mLin-10 is consistent with KIF17 and NR2B and was upregulated after TCI, and intrathecal administration of KIF17 antisense ODN suppressed the increase of mLin-10. When the analgesic effects disappeared, mLin-10 expression was again upregulated. This finding is consistent with previous studies which showed that: i) in the forebrain, overexpression of KIF17 increased the expression of mLin-10 and NR2B (24); ii) when KIF17 expression was blocked in hippocampal neurons, the expressions of mLin-10 and NR2B was also decreased (15). These results suggest KIF17, mLin-10 and NR2B may be a common adjustment, although we do not understand the specific mechanism involved in mLin-10 expression. However, these findings demonstrate that cargoes that contain NR2B are transported by KIF17, and mLin-10 is necessary for this function. Thus, in the spinal cord, KIF17 participated in NR2B transshipment through mLin-10 and plays an important role in the process of bone cancer pain in mice. KIF17 and mLin-10 may constitute a transfer complex protein (Fig. 6). In a subsequent study, we aim to show the specific mechanism of mLin-10 expression and whether other proteins exist that are involved in NR2B transport.

The present study contributes to the understanding of the mechanisms of bone cancer pain. In consideration of the finding that newly synthesized NR2B must be transported into dendrites and localized to synapses, the results may 
provide a new perspective on NR2B transport for clinical treatment.

\section{Acknowledgements}

This study was supported by the National Natural Science Foundation of China (nos. 81070892, 81171048, 81171047) and the Key Subject of Anesthesiology in the Jiangsu Province of China (XK201140).

\section{References}

1. Bray F, Jemal A, Grey N, Ferlay J and Forman D: Global cancer transitions according to the human development index (2008-2030): a population-based study. Lancet Oncol 13: 790-801, 2012.

2. Costantini M, Ripamonti C, Beccaro M, et al: Prevalence, distress, management, and relief of pain during the last 3 months of cancer patients' life. Results of an italian mortality follow-back survey. Ann Oncol 20: 729-735, 2009.

3. van den Beuken-van Everdingen MH, de Rijke JM, Kessels AG, Schouten HC, van Kleef $M$ and Patijn J: Prevalence of pain in patients with cancer: a systematic review of the past 40 years. Ann Oncol 18: 1437-1449, 2007.

4. Mantyh P: Bone cancer pain: causes, consequences, and therapeutic opportunities. Pain 154: S54-S62, 2013.

5. Goblirsch M, Zwolak P and Clohisy DR: Advances in understanding bone cancer pain. J Cell Biochem 96: 682-688, 2005.

6. Coleman RE: Clinical features of metastatic bone disease and risk of skeletal morbidity. Clin Cancer Res 12: 6243s-6249s, 2006.

7. Laurie DJ, Bartke I, Schoepfer R, Naujoks K and Seeburg PH: Regional, developmental and interspecies expression of the four NMDAR2 subunits, examined using monoclonal antibodies. Brain Res Mol Brain Res 51: 23-32, 1997.

8. Nagy GG, Watanabe M, Fukaya M and Todd AJ: Synaptic distribution of the NR1, NR2A and NR2B subunits of the $\mathrm{N}$-methyl-d-aspartate receptor in the rat lumbar spinal cord revealed with an antigen-unmasking technique. Eur J Neurosci 20: 3301-3312, 2004.

9. Klein T, Magerl W, Hanschmann A, Althaus $M$ and Treede RD: Antihyperalgesic and analgesic properties of the $\mathrm{N}$-methyl-D-aspartate (NMDA) receptor antagonist neramexane in a human surrogate model of neurogenic hyperalgesia. Eur J Pain 12: 17-29, 2008.

10. Qu XX,CaiJ,LiMJ,etal: Role of the spinal cord NR2B-containing NMDA receptors in the development of neuropathic pain. Exp Neurol 215: 298-307, 2009

11. Woolf CJ: Central sensitization: uncovering the relation between pain and plasticity. Anesthesiology 106: 864-867, 2007.
12. Gu X, Zhang J, Ma Z, et al: The role of N-methyl-D-aspartate receptor subunit NR2B in spinal cord in cancer pain. Eur J Pain 14: 496-502, 2010.

13. Paoletti P and Neyton J: NMDA receptor subunits: function and pharmacology. Curr Opin Pharmacol 7: 39-47, 2007.

14. Lau CG and Zukin RS: NMDA receptor trafficking in synaptic plasticity and neuropsychiatric disorders. Nat Rev Neurosci 8: 413-426, 2007.

15. Guillaud L, Setou M and Hirokawa N: KIF17 dynamics and regulation of NR2B trafficking in hippocampal neurons. J Neurosci 23: 131-140, 2003.

16. Hirokawa N, Niwa S and Tanaka Y: Molecular motors in neurons: transport mechanisms and roles in brain function, development, and disease. Neuron 68: 610-638, 2010.

17. Yin X, Takei Y, Kido MA and Hirokawa N: Molecular motor KIF17 is fundamental for memory and learning via differential support of synaptic NR2A/2B levels. Neuron 70: 310-325, 2011.

18. Schwei MJ, Honore P, Rogers SD, et al: Neurochemical and cellular reorganization of the spinal cord in a murine model of bone cancer pain. J Neurosci 19: 10886-10897, 1999.

19. Luger NM, Sabino MA, Schwei MJ, et al: Efficacy of systemic morphine suggests a fundamental difference in the mechanisms that generate bone cancer vs inflammatory pain. Pain 99: 397-406, 2002.

20. Chaplan SR, Bach FW, Pogrel JW, Chung JM and Yaksh TL: Quantitative assessment of tactile allodynia in the rat paw. J Neurosci Methods 53: 55-63, 1994

21. Wong-Riley MT and Besharse JC: The kinesin superfamily protein KIF17: one protein with many functions. Biomol Concepts 3: 267-282, 2012.

22 . Hirokawa N: Kinesin and dynein superfamily proteins and the mechanism of organelle transport. Science 279: 519-526, 1998.

23. Miki H, Okada Y and Hirokawa N: Analysis of the kinesin superfamily: insights into structure and function. Trends Cell Biol 15: 467-476, 2005.

24. Wong RW, Setou M, Teng J, Takei Y and Hirokawa N: Overexpression of motor protein KIF17 enhances spatial and working memory in transgenic mice. Proc Natl Acad Sci USA 99: 14500-14505, 2002.

25. Guillaud L, Wong R and Hirokawa N: Disruption of KIF17-Mint1 interaction by CaMKII-dependent phosphorylation: a molecular model of kinesin-cargo release. Nat Cell Biol 10: 19-29, 2008.

26. Setou M: Kinesin superfamily motor protein KIF17 and mLin-10 in NMDA receptor-containing vesicle transport. Science 288: 1796-1802, 2000.

27. Ho A, Morishita W, Hammer RE, Malenka RC and Sudhof TC: A role for Mints in transmitter release: Mint 1 knockout mice exhibit impaired GABAergic synaptic transmission. Proc Natl Acad Sci USA 100: 1409-1414, 2003.

28. Ni K, Zhou Y, Sun YE, Liu Y, Gu XP and Ma ZL: Intrathecal injection of selected peptide Myr-RC-13 attenuates bone cancer pain by inhibiting KIF17 and NR2B expression. Pharmacol Biochem Behav 122: 228-233, 2014. 